Arthroskopie $2020 \cdot 33: 65-66$

https://doi.org/10.1007/s00142-020-00352-8

(C) Springer Medizin Verlag GmbH, ein Teil von Springer Nature 2020

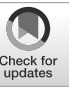

\author{
Michael T. Hirschmann ${ }^{1,2} \cdot$ Philipp Niemeyer ${ }^{3,4}$ \\ ' Department of Orthopaedic Surgery and Traumatology, Kantonsspital Baselland (Bruderholz, Liestal, \\ Laufen), Bruderholz, Schweiz \\ ${ }^{2}$ University of Basel, Basel, Schweiz \\ ${ }^{3}$ Orthopädische Chirurgie München (OCM), München, Deutschland \\ ${ }^{4}$ Klinik für Orthopädie und Unfallchirurgie, Universitätsklinikum Freiburg, Freiburg im Breisgau, \\ Deutschland
}

\title{
Fakten und Mythen der Zelltherapie in der Orthopädie
}

\section{Ein schmaler Grat zwischen experimenteller Medizin und wissenschaftlicher Evidenz}

logien und Verletzungen sehen. Auch das Internet ist voll von heilversprechenden Zelltherapie- oder Stammzellverfahren. Es scheint gerade so, als ob bereits heute die Möglichkeiten unbegrenzt wären. Einzelne Fälle werden als Beweis für das grenzenlose Potenzial der Zelltherapie herangezogen. Nicht selten wird z. B. eine Heilung der Arthrose, des Knorpelschadens, der Kreuzbandverletzung versprochen.

\section{》) Der Begriff der Stammzelle wird oft nicht korrekt verwendet}

Behandelte Patienten werden überzeugend in Testimonials zum Botschafter für den Erfolg der Zelltherapie gemacht. Es wird der Eindruck erweckt, dass mit der Zelltherapie schon heute medizinische Wunder möglich sind. Glaubt man den Proponenten, so wird eine einfache Injektion von spezialisierten Zellen eine Vielzahl von bisher unheilbaren Krankheiten therapieren können. Zudem wird von vielen Patienten in der Suche nach Alternativen zu einer Operation die Zelltherapie gerne angenommen, auch wenn diese nur als Selbstzahlerleistung verfügbar ist.

Des Weiteren wird der Begriff der Stammzelle oft nicht korrekt verwendet. Die meisten der angebotenen Therapien enthalten im engeren Sinn kaum oder keine Stammzellen. Gerade die aus körperei- genem Fett gewonnenen mesenchymalen Zellen werden oft synonym mit dem Begriff der Stammzelle verwendet. Dies ist jedoch eine Irreführung, da diese nicht wie aus Knochenmark gewonnene Zellen alle Eigenschaften einer Stammzelle aufweisen. Bei Stammzelle denkt jeder unweigerlich an die unglaublichen Fähigkeiten der Selbstheilung von Gewebe, und daher findet dieser Begriff weiterhin breite Verwendung. Der Grat zwischen Selbstmarketing bzw. Schamanentum und seriöser Medizin verschwimmt hier leider in zunehmendem Maße.

Dem gegenüber stehen aber zwischenzeitlich valide Studien, die den Nutzen von Zell- und Gewebeaufbereitungen belegen und Hinweise dafür geben, dass auch aus biologischer Sicht ein klinischer Effekt besteht, den es weiter zu untersuchen und analysieren gilt.

Ziel dieser Ausgabe der Arthroskopie ist es, für uns etwas Licht in das Dunkel der Diskussion um Stammzellen in der Orthopädie zu bringen. Der erste Teil beschäftigt sich daher mit den biologischen Grundlagen, die uns als Orthopäden oft nicht so vertraut und geläufig sind. Doch müssen wir in vermehrtem Maße auf Kongressen und auch in der täglichen Arbeit in Kontakt mit Vertretern von Firmen damit umgehen. Gerade hier ist sehr viel Halbwissen unterwegs, und manche unter uns mit etwas mehr Wissen zum Thema Stammzelle werden schnell zum Experten erhoben. unterschiedlicher orthopädischer Patho- 
Im ersten Artikel werden daher folgende Fragen beantwortet: Was definiert eine Stammzelle? Über welche Fähigkeiten muss eine Zelle verfügen, um als Stammzelle bezeichnet zu werden? Welche Zellen dürfen wir korrekterweise als Stammzellen bezeichnen?

In den weiteren Artikeln geht es um die regulatorischen Voraussetzungen für das Anwenden der verschiedenen Verfahren in der Zelltherapie in Deutschland, Österreich und der Schweiz.

Abschließend werden die Anwendungsmöglichkeiten der Zelltherapie und deren klinischen Ergebnisse in der Behandlung von orthopädischen Krankheitsbildern zusammenfassend dargestellt und diskutiert.

Michael T. Hirschmann

Philipp Niemeyer

\section{Korrespondenzadresse}

Prof. Dr. Michael T. Hirschmann

University of Basel

Basel, Schweiz

Michael.Hirschmann@unibas.ch

\section{Prof. Dr. Philipp Niemeyer}

Orthopädische Chirurgie München (OCM)

Steinerstraße 6, 81369 München, Deutschland

philipp.niemeyer@ocm-muenchen.de

Interessenkonflikt. M.T. Hirschmannund P. Niemeyer geben an, dass kein Interessenkonflikt besteht.
Weißer, C.

\section{Chirurgenlexikon}

2000 Persönlichkeiten aus der Geschichte der Chirurgie

Berlin Heidelberg: Springer-Verlag 2019, 1. Auflage, 501 S., 135 Abb., (ISBN: 978-3-662-59237-3), Hardcover 99,99 EUR

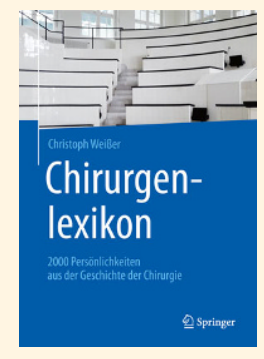

Beschäftigt man sich intensiver mit chirurgischen oder orthopädischen Fragestellungen, so bestätigt sich immer wieder das Gleichnis von den „Zwergen die auf den Schul-

tern von Riesen" sitzen. So rasch sich Medizin und Chirurgie gegenwärtig fortentwickeln, so sehr sind sie das Ergebnis eines jahrhundertelangen Erkenntnisprozesses. Die aktuelle chirurgische und orthopädische Praxis ist ohne die

die wissenschaftlichen Erkenntnisse und Erfahrungen früherer Chirurgengenerationen nicht zu verstehen.

Es ist das Verdienst des Würzburger Chirurgen und Oberarztes Christoph Weißer, ein umfassendes Lexikon zu wichtigen Persönlichkeiten der Chirurgie, Orthopädie und angrenzender Fachgebiete vorgelegt zu haben.

Der Autor informiert über die Lebensdaten, den beruflichen Werdegang und die Schwerpunkte der beruflichen Tätigkeit bedeutender Chirurgen. Weißer gelingt es dabei, die wesentlichen biographischen Aspekte knapp und verständlich darzustellen. Die Texte sind wissenschaftlich präzise, sie halten einer historischen Überprüfung stand und gehen weit über diejenigen Informationen hinaus, die bei einer Internetrecherche gewonnen werden können. Als Nachschlagewerk konzipiert, lädt es darüber hinaus zu einer spanenden und lehrreichen Lektüre ein. Wer wüsste nicht gerne mehr über Percival Pott, Bernhard Langenbeck, William A. Lane, Themistokles Gluck, Martin Kirschner, Albin Lambotte, K. H. Bauer, Martin Allgöwer und Klaus Klemm?

Der Autor erklärt die hauptsächlichen chirurgischen Eponyme und trägt somit zum
Verständnis von Begriffen bei, die zwar allgemein gebräuchlich sind, deren Kenntnis sich jedoch nicht unmittelbar erschließt. Weit über einhundert Abbildungen, zeitgenössische Zeichnungen und Fotografien, illustrieren den Band. Das Werk berücksichtigt einzelne Ärzte aus Antike und frühen Neuzeit, der Schwerpunkt liegt in dem Zeitraum vom 18. Jahrhundert bis in die Gegenwart. Berücksichtigt wurden die Lehrstuhlinhaber der chirurgischen Fächer im deutschsprachigen Raum, die Leiter der berufsgenossenschaftlichen Unfallkliniken sowie die Präsidenten der verschiedenen chirurgischen Fachgesellschaften.

Das umfassende Werk kann jedem Chirurgen und Orthopäden mit Nachdruck empfohlen werden.

K.-D. Thomann (Frankfurt am Main) 